\title{
When a patient's choices entail risks for others: third-party risks, relational ethics, and responsibilities of rehabilitation professionals
}

\section{Matthew Hunt, Stephen Clarke \& Raphael Lencucha}

To cite this article: Matthew Hunt, Stephen Clarke \& Raphael Lencucha (2019): When a patient's choices entail risks for others: third-party risks, relational ethics, and responsibilities of rehabilitation professionals, Disability and Rehabilitation, DOI: 10.1080/09638288.2019.1637950

To link to this article: https://doi.org/10.1080/09638288.2019.1637950

\section{曲 Published online: 31 Jul 2019.}

Submit your article to this journal $x$

\section{Џll Article views: 155}

\section{Q View related articles $\asymp$}

\section{View Crossmark data ¿}




\title{
When a patient's choices entail risks for others: third-party risks, relational ethics, and responsibilities of rehabilitation professionals
}

\author{
Matthew Hunt ${ }^{\mathrm{a}, \mathrm{b}}$, Stephen Clarke ${ }^{\mathrm{c}}$ and Raphael Lencucha ${ }^{\mathrm{a}, \mathrm{b}}$ \\ ${ }^{\mathrm{a} S}$ School of Physical and Occupational Therapy, McGill University, Montreal, Canada; ${ }^{\mathrm{b}}$ Center for Interdisciplinary Research in Rehabilitation, \\ Montreal, Canada; 'Biomedical Ethics Unit, McGill University, Montreal, Canada
}

\begin{abstract}
Purpose: Patients receiving rehabilitation care may act in ways that pose risks to the wellbeing of others. There are numerous situations when patients' actions might pose risks to others, including family members, other patients, or society. For example, such risks arise if patients wish to return home to living in an apartment but risk starting a fire while cooking, or if they insist on driving but their health condition makes doing so unsafe. These situations give rise to ethical challenges, including for health professionals as they seek to promote their patient's autonomy while minimizing risks for the patient and others.

Methods: We develop a conceptual analysis examining how relational ethics can inform understanding of rehabilitation professionals' responsibilities in responding to third-party risk.

Results: Relational ethics foregrounds the situated, dynamic, and interdependent nature of relationships between people, and supports dialogic approaches to clarify how best to respond. We present a set of 10 questions linked to the concepts of relational autonomy and relational social justice that can contribute to ethical reflection and deliberation among providers, patients, families, and others involved.

Conclusion: Relational ethics provides a distinctive angle of view on challenging issues of third-party risk in rehabilitation care.

> IMPLICATIONS FOR REHABILITATION

- Managing risk is a recurrent source of ethical challenge in rehabilitation care.

- Risks to third parties give rise to significant ethical tensions.

- We propose ten questions that can support a relational ethics approach for responding to situations of third-party risk.
\end{abstract}

\section{ARTICLE HISTORY}

Received 21 December 2018

Revised 21 June 2019

Accepted 26 June 2019

\section{KEYWORDS}

Clinical ethics; feminist ethics; personal autonomy; rehabilitation; risk; social justice

\section{Risks in the context of rehabilitation care}

Risks are an unavoidable facet of rehabilitation care. When participating in rehabilitation activities, patients risk disappointment, discomfort, and even physical harm (e.g., if a patient were to fall during therapy). The United Kingdom National Patient Safety Agency defines risk as "the probability that a specific adverse event will occur in a specific time period or as a result of a specific situation" [1, p.4]. Risk of physical harm is especially salient; however, risks relevant to rehabilitation care also include the potential for harm in social (e.g., strained relationships), emotional (e.g., feeling sad), or occupational (e.g., being unable to participate in a valued work or leisure activity) dimensions. In turn, healthcare organizations and health professionals have obligations to identify, manage, and mitigate risks.

Managing and mitigating risk has important ethical implications across healthcare settings [2]. To begin, there is a degree of vulnerability inherent to professional-patient relationships, and asymmetries of power. Health professionals have responsibilities toward the safety and well-being of their patients, and to respect and support their capacity for making informed decisions. They also have power to shape certain aspects of their patients' lives (though this influence will vary according to setting, e.g., more during in-patient rehabilitation care and much less for homecare).
At the same time, patients have important rights, including in most cases, the right to make and enact autonomous decisions consistent with their values. Given the ethical complexity of risk management in professional-patient relationships, it is important to consider the meaning and scope of professional and social responsibility in the context of rehabilitation care. How ought rehabilitation professionals respond to different situations of risk?

In the rehabilitation literature, discussions of risk and professional responsibility have considered potential physical and emotional harms that may be experienced by professionals who are placed in situations involving challenging, combative patients [3]. Another category of risk-related encounters centers on decisions by patients to act in ways that present threats to their own health while receiving rehabilitation care. For example, a patient in an in-patient rehabilitation unit who, following a stroke, experiences dysphagia yet refuses to follow the modified diet that has been prescribed to prevent aspiration [4]. When patients place their own safety at risk (and in ways not aligned with the goals of therapy), healthcare professionals may struggle to navigate the dual aims of respect for patient autonomy and protection of their patients' well-being [5,6]. Less attention has been given to thirdparty risk. Third-party risk emerges when the decisions made by a person have potential consequences for others (e.g., other 




Figure 1. Resolving dilemmas and responding to ethical tensions.

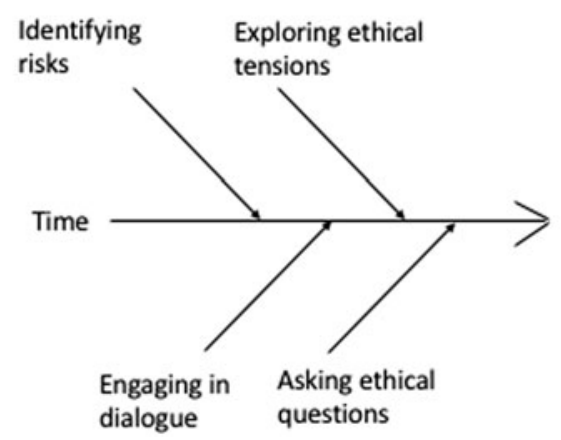

patients, family members, neighbors, or community members). Some discussion has occurred in relation to health-related driving risks [7] and disruptive behaviors in rehabilitation facilities [8]. These commentators describe scenarios where patient choices have the potential to place third-party stakeholders at risk of harm, and report that rehabilitation professionals experience these situations as ethically challenging.

Our aim in this article is to critically appraise possibilities for navigating the ethical terrain of third-party risk in rehabilitation. We present and discuss how a relational ethics framework can help orient rehabilitation professionals when responding to ethically challenging situations involving risks to third parties. We draw upon the concepts of relational autonomy and relational social justice to structure our analysis. We then introduce three cases that involve third-party risks and illustrate the layers of overlapping ethical considerations involved in these situations, discussing how they pose challenges for rehabilitation professionals and for theories of healthcare ethics. Examining these cases through a relational lens, we introduce 10 questions that can support reflection and deliberation in situations involving third-party risks. Engaging with these questions can help rehabilitation professionals attend to relational and structural features of a situation and clarify the scope and reach of their ethical responsibilities, while encouraging deliberation with patients, colleagues, and others to address salient ethical considerations. We do not analyze the legal dimensions of third-party risk, though we acknowledge important legal considerations related to confidentiality and the duty to warn. While our focus here is on third-party risks, a relational ethics approach is very relevant for considering risk in rehabilitation more broadly [6], as well as other sources of ethical concern.

\section{Relational ethics}

Ethical analysis in healthcare has often focused on identifying the "right" approach for making contested or controversial decisions, including situations of ethical dilemma. Pincoffs has described such an emphasis as quandary ethics [9]. In medical ethics, this has often been addressed by assessing how key principles apply to the situation, including respect for autonomy, beneficence, non-maleficence, and justice [10]. Articulations of principles are understood to act "as guidelines that are interpreted and made specific for policy and clinical decision-making" [11, p.84]. A relational approach to ethics also engages with ethical principles but emphasizes the need for ongoing attentiveness to how values are being enacted or thwarted, and how communication and collaboration can be promoted over time. It also foregrounds the situated, dynamic, and interdependent nature of relationships between individuals. Particularly salient for healthcare, this perspective reframes "decisions for," as "decisions with" [12]. The cornerstone of relational ethics is that decisions are always made in the context of interpersonal relationships. In healthcare, ethical decisions are inherently enmeshed in complex social relationships and patterns of interdependency. The actions of healthcare professionals and patients, as well as families, may also extend beyond the immediate clinical relationship (e.g., for other patients, colleagues, institutions, the public). Relational ethics thus provides a valuable perspective for addressing responsibilities of rehabilitation health professionals in situations where patients wish to enact choices that have the potential to create or heighten risks for others [6].

Austin et al. [13] have described relational ethics as an action ethic, which places a priority on the moral requirements involved in respecting particular others with whom we enter into relationships. Relational ethics' consideration of a wider sets of relationships, including relationships that patients have with families, friends, colleagues, and neighbors, or even society more generally, make it particularly relevant for considering situations of thirdparty risk. Instead of seeing third-party risk as a dilemma to be resolved, a relational approach will conceive of a process of engagement and interaction over time (Figure 1). From a relational ethics perspective, situations that involve risk to third parties are understood as characterized by ethical tensions that must be grappled with and worked through, rather than as a dilemma requiring tradeoffs among competing principles. Working collaboratively, seeking to understand different perspectives and asking questions can all help health professionals to apply a relational approach.

Kenny, Sherwin, and Baylis [14] suggest that a relational approach is preferable to individual-focused paradigms of ethical decision-making when there are consequences that extend beyond patient-professional relationships. They point to two key relational concepts that we take up in our analysis: relational autonomy and relational social justice. Before discussing these concepts and considering their application for third-party risks, we present three illustrative case studies of scenarios in which patients' actions and choices are associated with risks to third parties.

\section{Three case studies}

- $\quad$ Ms A is a 56-year-old woman who lives in a long-term care facility. She uses a motorized wheelchair to move about the institution, and enjoys socializing with other residents. In recent months, Ms $\mathrm{A}$ has been driving her wheelchair more 
Table 1. Questions to stimulate reflection and deliberation about situations of third-party risks.

Questions through the lens of relational autonomy

1. What is the quality of the therapeutic relationship and does it foster shared decision-making?

2. What is the nature (probability and gravity) of the risk?

3. How are these risks perceived from different perspectives and how can mutual understanding be fostered?

4. What efforts have been made to support communication around these risks and to raise awareness of the implications for other people?

5. What concrete steps have been taken to preserve the patient's sense of autonomy?

6 . What role can the people closest to the patient play in the situation?

7. What ought to be done to uphold confidentiality and what are the limits of confidentiality in this situation?

Questions through the lens of relational social justice

8. What are the social conditions that shape the patient's ability to make decisions, their empowerment as a moral agent, and how they perceive risks?

9. Are there social stigmas or barriers that contribute to the situation?

10. How can the patient's meaningful participation in society be preserved in a way that mitigates risk for others?

quickly and sometimes veers close to other residents or objects in the environment. Staff members, as well as other residents, have expressed concerns regarding the safety of people walking in the hallways. This concern is particularly acute for residents who are frail, less steady on their feet, or who lack agility to get out of the way of Ms A's wheelchair. This issue has been raised with Ms $A$ but she has not altered how she uses her wheelchair, saying that she does not see why anyone is concerned and she has the right to use her wheelchair in any way she wishes. The motorized wheelchair is the only means by which Ms A can move about and socialize. It is crucial for her independence. The situation is a source of anxiety for some facility residents who appear more likely to stay in their rooms and avoid walking in the halls for fear of being knocked over.

- $\mathrm{Mr} \mathrm{B}$ is a 76-year-old retiree who experienced a lacunar stroke three years ago. He lives by himself and receives home care, including physical and occupational therapy at his condo in a large, accessible downtown apartment building. Recently, the occupational therapist has noticed that $\mathrm{Mr}$ $B$ is showing signs of confusion and memory loss. He frequently forgets to complete simple tasks. Mr B's daughter told the occupational therapist that when she last visited, her father had left a pot on the stove with the element on, and it had boiled dry. Mr B has always valued his independence and is reluctant to accept support from others. He has refused to move to an assisted living center where more assistance is available. Discussing these concerns with $\mathrm{Mr} B$ is made more difficult as he gets frustrated and angry whenever he feels his memory and judgment are being questioned. Mr B's children, as well as the occupational therapist, are worried that Mr. B might start a fire while cooking which would result in harm for himself and his neighbors.

- Mrs C is a 44-year-old woman who was struck by a car six months ago. She had multiple fractures and a traumatic brain injury. She is nearing discharge from the in-patient unit of a rehabilitation hospital and will return to the home which she shares with her husband and teenage daughter. Mrs $C$ has progressed well in the rehabilitation program and is eager to resume her everyday activities. She continues to demonstrate a lack of insight, however, and appears to have difficulty understanding the consequences of her decisions. In the course of making discharge plans, she tells the social worker that she intends to resume her work as a school crossing guard immediately upon her discharge. She explains that she loves her job and that her salary is important for her family's finances which have been strained over the course of her rehabilitation.

\section{Responding to third-party risks in rehabilitation: The contribution of relational ethics}

The roles and engagement of the rehabilitation professionals in the three case studies raise questions about the extent of their responsibilities toward the specific patient, as well as toward other individuals-be them other residents in a care facility (in the case of $\mathrm{Ms} \mathrm{A}$ ), neighbors of a patient receiving homecare (in the case of $\mathrm{Mr} \mathrm{B}$ ), or children who use the school crossing where a patient recovering from an accident may be posted as a crossing guard upon her discharge from the rehabilitation hospital (in the case of Mrs C). While the focus of our analysis is on the responsibilities of rehabilitation professionals, these cases demonstrate how situations of third-party risk can give rise to ethical uncertainty and tension among the ethical commitments of all those involved.

We have suggested that relational ethics can help orient rehabilitation professionals' responses to situations involving third-party risk in rehabilitation. This approach is well suited to generating questions, rather than prescriptions, to inspire and guide ethical deliberation. Therefore, as we discuss how relational autonomy and relational social justice relate to third-party risks, we will introduce a series of questions that aim to foster reflection and dialog. These questions are presented in Table 1. The questions are intended to promote careful consideration of a situation from different perspectives while being attentive to relationships, forms of responsibilities, and sources of structural vulnerability. The questions can serve to spark reflection for individuals or teams, and to ensure that a wide range of ethical considerations are taken into account.

Rehabilitation professionals may use the questions to inform and inspire discussion with their patients and (possibly) their family or others close to them. The questions can also be used during team meetings or rounds to guide discussion. We developed these questions with situations of third-party risk in mind. However, they have broader relevance. The questions could also be used to reflect on situations where there is risk of harm for the patient herself or himself, or adapted for other situations of ethical uncertainty in rehabilitation care.

\section{Relational autonomy}

Respect for autonomy is a central ethical value in healthcare $[15,16]$. It is instantiated in practices such as informed consent and reflected in legal parameters of healthcare practice. Autonomy has generally been conceived as a person's right to self-govern, with a corollary negative duty on others not to interfere with the practice of others' autonomous decisions without 
compelling reasons for doing so. This notion of autonomy is an individualistic one. In recent decades, a relational view of autonomy has been advanced, one that foregrounds relationships and interdependence. Respecting relational autonomy requires not just refraining from interfering with a person's ability to act from their own interests, but also seeking opportunities to uphold, support, and expand these capacities [17]. It maintains consideration of patient autonomy, and other important individual rights, while recognizing that patients are socially, politically, and economically situated. One implication of this understanding is identified by Kenny et al. [14] who argue that the most effective means to promote autonomy is sometimes through change to an individuals' social situation, rather than simply protecting their freedom to act however they choose.

From a relational perspective, autonomy is multidirectional. The capacity for a patient to, for example, return home or resume their employment after experiencing an illness or injury is enabled by the community and institutions that make these options possible. In other words, autonomy is not simply given or taken away, respected, or superseded by other concerns. Instead, it is built and sustained by the patient's relationships with others, the caring labor of healthcare professionals and other caretakers, and the willingness and capacity of the patient to act according to her or his own interests. This notion of autonomy also recognizes that the same conditions that foster individual autonomy must be considered for others in the person's social environment. For example, do the conditions that foster autonomy for a patient also foster autonomy for others? Or, do a patient's autonomous actions impact the autonomy of others? From this perspective, respect for autonomy is more complex and nuanced. For example, a rehabilitation professional might focus on maintaining the patient's sense of autonomy by enabling the pursuit of her or his interests while emphasizing certain values, such as not impinging on the autonomy of others, maintaining open lines of communication, and recognizing the patient's need to be listened to. This notion of autonomy is what Stirrat and Gill [16] refer to as "principled" autonomy, where an autonomous individual (a patient in this case) is one "who has the capacity to make a settled choice about medical interventions on themselves, to do so responsibly in a manner considerate to others" (emphasis added, p.103). It also recognizes that a person's autonomy can be expressed and fostered in more than one way, and that helping someone imagine alternate possibilities is a means of advancing their autonomy. Given the potential for harm to self and others, a relational view of autonomy makes it more than merely a right that is either protected or transgressed, but also a capacity to be situated in the broader relationships that exist in the patient's social environment. The following seven questions are informed by this concept of relational autonomy.

\section{Questions through the lens of relational autonomy}

What is the quality of the therapeutic relationship(s) and does it foster shared decision-making?

Relational autonomy places moral value on involving and foregrounding the web of social relationships of patients. This notion focuses attention on how autonomy is enabled through relationships. When facing a situation of third-party risk, a starting place for health professionals can be to consider the quality of the therapeutic relationship that exists between them and the patient, as well as with others close to the patient. For example, if the team working with Ms A were to only take account of the risks to other residents, without considering what is at stake for Ms A and the quality of their relationships with her, they may quickly alienate her and undermine the possibility of working collaboratively. By carefully considering their relationship with Ms $A$, they can explore the level of trust, vulnerability, openness, sensitivity, and other facets of the relationship. In particular, this question may orient professionals to reflect on whether or not these relations foster open discussion with Ms A and the potential for shared decision-making, and how this might be enhanced. The insight provided by relational ethics here is the importance of beginning with a dialogic approach that engages with her autonomy to make decisions that have bearing on her well-being, but also on the safety and well-being of others.

\section{What is the nature (probability and gravity) of the risk?}

Many risks associated with healthcare interventions are well known (e.g., medication side effects). Other risks in rehabilitation care, such as those raised in the three case studies, are difficult to measure. Acknowledging uncertainty related to risk estimation is thus crucial. While it is important not to dismiss risks whose likelihood or gravity is difficult to gauge, another concern is to avoid overstating risks. Taking risks seriously in the light of relational ethics should entail using tools and resources that are available to carefully analyze the risk of harms rather than making assumptions about them (e.g., evaluating the likelihood that Mr B could start a fire). Rehabilitation professionals can utilize many sources of information to evaluate risks, including ecological assessments (e.g., an occupational therapist could assess risks associated with Mrs C's work plans) for the patient and others involved. This might include considering how the patient, the patient's family, the care team, and the general public will each be affected by a continued situation of risk, and by any measures that are proposed to mitigate it. This information is important not only for the healthcare team but also for the patient (and, potentially, their family and loved ones) to help them make well-considered decisions.

How are these risks perceived from different perspectives and how can mutual understanding be fostered?

There is a tendency to approach risk management through a technical or reductionistic decision-making process whereby the emphasis on the identified risk supersedes or crowds out questions of values, meaning, and other social considerations. Health professionals rely on clinical evaluations, as well as their own experience and professional knowledge to identify and estimate potential risks. However, differing perspectives between professionals and patients, family members, and others affected by risky behavior can inform a more responsive approach to risk management. For example, it is important for health professionals to understand the meaning that a particular choice has for their patient. In this way, cooking may be particularly meaningful for $\mathrm{Mr} \mathrm{B}$ as it allows him to prepare foods that are familiar and comforting, based on family recipes and continuous with his past. Understanding what lies behind Mr B's stated preferences is thus a key for health professionals to orient their response to the situation. Likewise, health professionals should seek to make clear the rationale behind the interventions that they propose. For example, suggesting a shut-off timer on Mr B's stove may be perceived as a heavy-handed restriction of his autonomy but through discussion might be understood as a reasonable compromise so that $\mathrm{Mr} \mathrm{B}$ can continue to cook without placing himself and others at risk. 
What efforts have been made to support communication around these risks and to raise awareness of the implications for other people?

It is possible that patients and/or their families may not appreciate how their actions could impact others. Candid discussion of risks and potential consequences is thus crucial as a means of supporting patient autonomy so that they better understand what is at stake. Evidence suggests that improved understanding of how hardship or suffering is experienced by others will lead to greater "other-mindedness" and altruistic behavior $[18,19]$. The assumption for the relational ethics of third-party risk is that when rehabilitation professionals communicate information about how a patient's decisions may affect others, this knowledge may serve to inform dialog about ways to avoid or mitigate these effects. As noted above, this approach will be strengthened when risks have been evaluated and, where possible, measured. Rehabilitation professionals, however, should strive to avoid situations where patients feel shame or guilt. For example, in the case of Ms A, health professionals should carefully consider how to discuss with her the concerns expressed by other residents of the home regarding their safety in the hallways. In some circumstances, they may judge that it is better to refrain from mentioning these concerns.

What concrete steps have been taken to preserve the patient's sense of autonomy?

A commitment to relational autonomy recognizes that a person's autonomy is contingent on the relational, social, and institutional factors that enable them to act according to their interests. From this perspective, health professionals have a moral responsibility to use their position of knowledge and institutional power to help patients maintain their dignity. In the case of Mrs C, if risks are confirmed by the ecological evaluation, the social worker could support Mrs C to identify alternative employment or volunteer options. As Kenny et al. [14] argue, it is possible that a patient's sense of autonomy can be improved by finding themselves in an improved social situation. A key challenge with these types of third-party risk scenarios is that by attending to the risk to third parties, there may be new concomitant risk to the patient, such as the risk of losing employment, or the ability to maintain relationships with others. The approach that we are advancing is to move beyond rigid individualism or collectivism to consider how those working in healthcare settings can foster individual autonomy and dignity while working to mitigate harm to others through dialog. These risks must be acknowledged and weighed as the rehabilitation professional seeks to work collaboratively with their patient to identify solutions.

What role can the people closest to the patient play in the situation?

When information and dialog does not lead to acceptable solutions from the patient's perspective, it may be appropriate to involve family members and other close personal relationships. Making connections to the people who are closest to the patient can be effective for communicating with patients, and for gaining deeper understanding of the patient's social reality. However, respecting relational autonomy requires involving persons with whom the patient is comfortable, and only when he or she provides permission to do so. A relational perspective does not involve circumventing the patient's own wishes and preferences. However, family members might well have creative solutions to a complicated problem, insight into the patient's social context and personal history, and may be a source of support for mitigating future risk. There may be a wider array of options available and the healthcare professional may feel empowered to suggest more creative solutions if they know that the patient has close family members or friends who are engaged and supportive.

What ought to be done to uphold confidentiality and what are the limits of confidentiality in this situation?

In some cases of third-party risk, health professionals may be faced with the dilemma of protecting their patient's confidentiality while seeking to protect the patient and/or others from harm. This dilemma is heightened when risks are evaluated as being more grave, likely, and imminent. There are legal considerations to such situations that are beyond the scope of our analysis. However, these considerations require careful attention in light of the laws and professional norms in a particular jurisdiction. Generally, health professionals are bound to protect their patient's privacy unless a person's actions present a clear and imminent danger of death or severe bodily harm to themselves or others. In all cases, estimation of risk will be crucial, such as in the case of Mrs C. Thus far, we have suggested that open communication and inclusion of stakeholders guides a relational approach to third-party risks. However, this demands equal attentiveness to the patient's legal and ethical right to privacy in taking such a relational approach, and, potentially, to legal and professional responsibilities of health professionals related to the duty to warn in specific situations.

\section{Relational social justice}

Justice as a principle in healthcare ethics typically refers to concerns that resources are distributed equitably, and that care is reasonably accessible and provided in a nondiscriminatory manner [10]. Questions pertaining to social justice also come into focus from a relational perspective [14]. Social justice involves the recognition that social structures and attitudes can have a detrimental effect on the wellbeing of individuals who are differently situated (e.g., more or less privileged, wealthy, or socially connected) and "stresses the fair disbursement of common benefits and sharing of common burdens" [20, p.1053]. A relational perspective seeks to understand and address how disadvantaged and disenfranchised groups are disproportionately denied access to various kinds of social goods. It also draws attention to how social structures contribute to making many ethically challenging situations more difficult to resolve, including situations of risk for both patients and third parties. A related concept in the field of occupational science is occupational justice. Occupational justice recognizes that societal structures are implicated in both fostering and hindering one's engagement in activity that is meaningful and fulfilling [21]. For its part, relational social justice expands the scope of ethical concern beyond the relationship between the healthcare professional and patient, and even for the organization of health services within particular institutions, taking into account broader social structures, power dynamics, and various forms of marginalization related to disability, socioeconomic status, language, gender, and race, among others.

\section{Questions through the lens of relational social justice}

What are the social conditions that shape the patient's ability to make decisions, their empowerment as a moral agent, and how they perceive risks?

Risks may present differently based on the systemic challenges faced by a patient. For example, a patient who faces language 
barriers may feel less empowered to make decisions or communicate their needs. Systemic disadvantages may make some patients less able to make choices and disempowered in their own decision-making capacity. They may also lead some individuals to make a risky choice due to limited options available to them as a result of structural features. For example, financial constraints for Mrs C may lead her to resume work earlier than she would otherwise choose because she feels strong economic pressure to do so, or Mr B may face barriers to accessing support for meal preparation due to rules and regulations in the building where he lives. Identifying such considerations will help rehabilitation professionals to better understand the patient's situation and can open up opportunities to address these structural features.

Are there social stigmas or barriers that contribute to the situation?

A particular structural feature that warrants attention is the role of stigma, including a person's perspective of how others may view her or him. Patients may experience stigma, or feelings of shame, associated with a lack of independence, and the pressure to remain independent (i.e., driving, cooking, working) may keep them from recognizing and mitigating third-party risks. Rehabilitation professionals should also consider how their actions might contribute to marginalizing patients from their social communities. In the case of Mrs C, concerns for the health and wellbeing of the children using the crosswalk are central. However, concerns for occupational justice may also weigh heavily on the rehabilitation professional, including the patient's need for employment (and the resources employment provides), along with recognizing the potential for community building that meaningful employment gives. Rehabilitation professionals may recognize that Mrs C's lack of employment and lack of income could lead to socio-economic barriers and social stigma. Applying a relational ethics approach requires considering that discouraging Mrs $C$ from resuming her previous work due to the risk of harm to others, may have negative consequences for her. This perspective also provides an orientation to examine what Mrs $C$ finds meaningful in her employment, and through this dialog the health professional may be able to work with her to find equally meaningful employment opportunities that pose less risk to others.

How can the patient's meaningful participation in society be preserved in ways that mitigate risk for others?

At times, risky behavior may lead to disruption in a patient's social, emotional, or economic stability. A change or rupture in one's meaningful daily activities, whether through work, mobility in one's community, or cooking for oneself, can have important implications for the wellbeing of an individual. Given the risks involved, it seems prudent for the rehabilitation team to encourage or seek to persuade Mrs $C$ to not return to her position until her cognitive deficits have resolved sufficiently or additional supports implemented for her to perform her work duties safely. However, they should also consider alternate solutions for Mrs C due to the potential financial and occupational costs. This consideration also returns to earlier discussion of the need to identify the meaningful aspects of the activity. For Mrs C, it may be the sense of service to her community rather than the particular type of job that is meaningful to her. In this case, the rehabilitation professionals can work with Mrs $C$ to find other avenues of service that do not present risk to others. These avenues can incorporate her need to generate an income for herself and her family.

\section{Conclusion}

Situations when a patient's choice and actions could result in harmful consequences for other people present unique ethical challenges for rehabilitation professionals. Such situations raise questions about the nature and scope of professional responsibility. As we have demonstrated in this article, a relational ethics approach can usefully illuminate important dimensions of these often vexing situations. A relational approach is well suited to an iterative process of asking questions to guide dialog to draw attention to ethically significant features of a situation, and to help clarify how best to manage and respond to risk. This approach encourages rehabilitation professionals to extend their ethical gaze beyond proximal risks to also consider the broader social and relational implications of individual choices and actions, particularly when the potential consequences extend beyond the boundaries of the patient-professional relationship. The ultimate aim of this article is to stimulate engagement and an informed dialog by attending to salient features of particular situations of third-party risk.

\section{Acknowledgments}

The authors are grateful to members of the Global Health, Ethics and Rehabilitation lab group for their helpful comments on an earlier version of this article.

\section{Disclosure statement}

The authors report no declarations of interest.

\section{Funding}

Matthew Hunt and Raphael Lencucha are supported by Research Scholar Awards from the Fonds de recherche du Québec - Santé.

\section{References}

[1] National Patient Safety Agency (NPSA). Healthcare risk assessment made easy. London: National Health Service; 2007.

[2] Runciman B, Merry A, Walton M. Safety and ethics in healthcare: a guide to getting it right. London: CRC Press; 2017.

[3] Glancy GD, Chaimowitz G. The clinical use of risk assessment. Can J Psychiatry. 2005;50:12-17.

[4] Kaizer F, Spiridigliozzi AM, Hunt MR. Promoting shared decision-making in rehabilitation: development of a framework for situations when patients with dysphagia refuse diet modification recommended by the treating team. Dysphagia. 2012;27:81-87.

[5] Browne A. Helping residents live at risk. Camb Q Healthc Ethics. 2003;12:83-90.

[6] Hunt MR, Ells C. Partners towards autonomy: risky choices and relational autonomy in rehabilitation care. Disabil Rehabil. 2011;33:961-967.

[7] Mazer B, Laliberté $M$, Hunt $M$, et al. Ethics of clinical decision-making for older drivers: reporting health-related driving risk. Can J Aging. 2016;35:69-80.

[8] Young JM, Sullivan WJ. Ethical concerns of staff in a rehabilitation center. HEC Forum. 2001;13:361-367. 
[9] Ellis C. Telling secrets, revealing lives: relational ethics in research with intimate others. Qual Inq. 2007;13:3-29.

[10] Austin W, Bergum V, Dossetor J. Relational ethics: an action ethic as a foundation for health care. In: Tschudin V, editor. Approaches to ethics: nursing beyond boundaries. New York (NY): Butterworth-Heinemann; 2003. p. 45-52.

[11] Pincoffs E. Quandary ethics. Mind. 1971;80:552-571.

[12] Beauchamp TL, Childress JF. Principles of Biomedical Ethics. New York (NY): Oxford University Press; 2013.

[13] Beauchamp TL. Principlism and its alleged competitors. Kennedy Inst Ethics J. 1995;5:181-198.

[14] Kenny NP, Sherwin SB, Baylis FE. Re-visioning public health ethics: a relational perspective. Can J Public Health. 2010; 101:9-11.

[15] Gillon R. Ethics needs principles-four can encompass the rest-and respect for autonomy should be "first among equals". J Med Ethics. 2003;29:307-312.
[16] Stirrat GM, Gill R. Autonomy in medical ethics after O'Neill. J Med Ethics. 2005;31:127-130.

[17] Sherwin S. A relational approach to autonomy in health care. In: Gedge E, Wluchow WJ, editors. Readings in health care ethics. Peterborough (ON): Broadview Press; 2012. p. 14-32.

[18] De Waal FB. Putting the altruism back into altruism: the evolution of empathy. Annu Rev Psychol. 2008;59: 279-300.

[19] Van Lange PA. Does empathy trigger only altruistic motivation? How about selflessness or justice? Emotion. 2008;8:766.

[20] Gostin LO, Powers M. What does social justice require for the public's health? Public health ethics and policy imperatives. Health Affairs. 2006;25:1053-1060.

[21] Durocher E, Gibson BE, Rappolt S. Occupational justice: a conceptual review. J Occup Sci. 2014;21:418-430. 